\section{CANCER}

\section{Silent cancer suppression}

A recent study in Nature uncovers new functions of BRCA1 that may underlie tumorigenesis in BRCA1-deficient individuals.

BRCA1 is a well-known tumor suppressor, and its loss is a common driver of familial breast and ovarian cancer. BRCA1 activities in normal cells lie at the core of the cellular pathways that maintain genomic integrity throughout DNA replication and in response to stress. But how its disparate functions contribute to its tumor suppressor role is just starting to be elucidated.

Zhu et al. (doi:10.1038/nature10371, 8 September) observed that BRCA1 deficiency led to chromatin disorganization at heterochromatic centers where noncoding, potentially harmful DNA repeat sequences, called satellites, are tightly packed away. Heterochromatin silences the expression of satellite repeats, and the authors found that BRCA1's histonemodifying activity is important for the gagging function of the heterochromatic structures. In the absence of BRCA1, the normally silent satellites are expressed and can run amok, causing genomic instability.

The authors also show that deregulated expression of heterochromatic repeats is present in human breast tumors and that expression of satellite repeats in mammary epithelial cells can drive the initial stages of malignant cellular transformation. Thus, this work not only provides a new view of how BRCA1 deficiency can lead to tumor formation but also highlights the contribution of seemingly inert DNA repeats to tumorigenesis. - VA

\section{NEUROSCIENCE}

\section{Stressed out?}

The negative effects of stress on behavior are familiar to many of us. A recent study in Neuron (71, 498-511) discloses a possible molecular mechanism to account for them.

Experimenting on mice, Michael Bruchas et al. focused on the serotonergic neurons of the dorsal raphe nuclei-a brain region crucial for mood-related behaviors. The team selectively knocked out the $\alpha$ isoform of p38 mitogen-activated protein kinase (MAPK)—which regulates stress-dependent responses-from raphe neurons and found that the mutant mice were more resilient to stress. So, whereas wild-type mice showed depression and increased drug seeking in response to stress, the mice lacking p38 $\alpha$ kept their cool in the face of adversity.

Mechanistically, stress induced p38 $\alpha$ acti-

INFECTION

\title{
Slow progress for malaria vaccines
}

Malaria kills one million individuals each year, and half the world's population is at risk of infection. Yet there exists no effective vaccine for this disease. Two recent clinical studies of candidate malaria vaccines reflect the ongoing challenges to developing a protective vaccine (Science doi: $10.1126 /$ science. 1211548,8 September; N. Engl. J. Med. 365, 1004-1013).

Epstein et al. tested in humans the protective efficacy of a vaccine

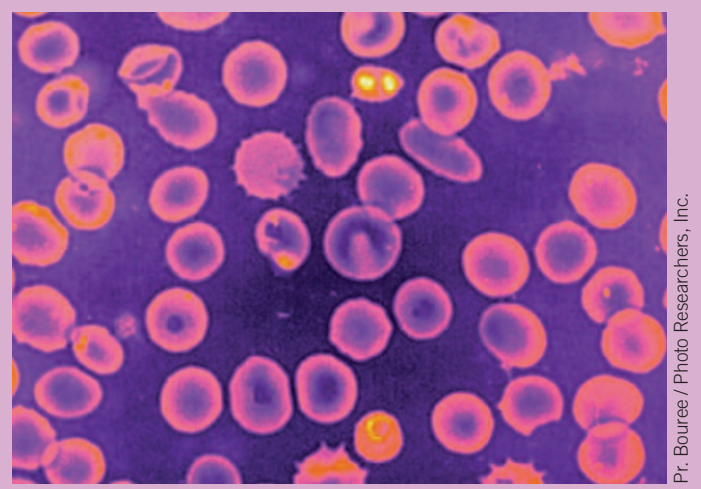
comprised of live attenuated but nonreplicating Plasmodium falciparum sporozoites injected subcutaneously or intradermally. But of 44 immunized individuals, only two were protected against challenge with $P$. falciparum-infected mosquitoes. However, intravenous injection of a similar vaccine in mice protected $71-100 \%$ of mice from challenge with the mouse parasite Plasmodium yoelii. The authors suggest that the route of administration may be crucial to the efficacy of their vaccine, and a further clinical trial will test this hypothesis in humans by assessing protection conferred by intravenous injection.

Whereas Epstein et al. tested whether the whole parasite could induce protection against infection, Thera et al. reported the results of a double-blind randomized trial of a subunitor protein-vaccine designed to reduce the clinical severity caused by Plasmodium infection of red blood cells. But of $\mathbf{4 0 0}$ children immunized with this vaccine or a control rabies vaccine, only $17.4 \%$ of the recipients of the subunit vaccine were protected from clinical malaria at 6 months.

The malaria life cycle is extremely complex, and the mechanisms required for vaccineinduced protective immunity in humans remain unclear. Although clinical trials of one vaccine candidate-RTS,S-are quite advanced and the results are promising, protection from infection is not complete, and development of additional vaccine candidates that elicit significant protection and can be feasibly administered to at-risk populations is essential. $-A F$

vation, which caused serotonin transporters to translocate to the membrane and speed up the clearance of serotonin from the synapse. In mutant mice, serotonin uptake was reduced, leading to a behavioral effect that shares some similarities to the effects of the very popular antidepressants known as selective serotonin uptake inhibitors. - JCL

\section{METABOLISM}

\section{A lipid link for Parkin}

It has been suggested that dysregulation of lipid biology may contribute to pathology in neurodegenerative diseases. A new paper now indicates that further study of this possibility would be warranted in Parkinson's disease by showing that the protein Parkin, which is mutated in some people with Parkinson's disease, regulates lipid metabolism.

On the basis of epidemiological evidence and preliminary observations in mice lacking
Parkin, Kye-Young Kim et al. (J. Clin. Invest. 121, 3701-3712) hypothesized that Parkin may be involved in the regulation of lipid metabolism. To test this, they compared wildtype and Parkin-knockout mice fed a high-fat diet (HFD) and found that the mice lacking Parkin were resistant to HFD-associated metabolic changes, including weight gain and hepatic insulin resistance. Moreover, the Parkin knockouts did not induce the expression of lipid transport proteins observed in the livers of wild-type mice on an HFD.

By studying hepatocytes overexpressing Parkin as well as mouse cells and cells from people with Parkinson's disease lacking Parkin, the authors showed that, mechanistically, Parkin mediates its effects on fat uptake by stabilizing the lipid transport protein CD36. However, whether defects in lipid metabolism contribute to Parkinson's disease pathogenesis in people with mutations in PARK2, the gene encoding Parkin, remains to be investigated in future studies. $-M S$ 